\title{
A MORPHOLOGICAL COMPARISON OF FOVEAL DEVELOPMENT IN MAN AND MONKEY
}

\author{
ANITA HENDRICKSON \\ Seattle, Washington
}

\section{SUMMARY}

The fovea can first be identified in both monkey and human retina at $26-30 \%$ gestation as a region containing all adult retinal layers and only cone photoreceptors. A shallow foveal pit and cone outer segments appear by 63-65\% gestation in both species. Prenatal development continues rapidly in the monkey, so that by birth a single layer of inner retinal neurons are present in the fovea, cones are three cells deep, inner segments are elongated, and outer segments are up to $50 \%$ of inner segment length. In contrast, human fovea does not reach a similar stage until several months after birth. The fovea is adultlike in monkey at 12 weeks and in human at 11-15 months, although human will mature further up to four to five years. This study shows that human fovea is less mature at birth than monkey but develops rapidly in infancy, suggesting that it may be even more susceptible to postnatal environmental influences than the commonly-used monkey model.

The fovea is a specialised region which lies at the centre of gaze in the primate retina. The inner retinal layers are absent, creating a foveal pit, although the region just surrounding the pit, called the foveal slope, contains the highest number of ganglion cells (GC) in the retina (rev. in 1). The foveal centre, called the foveola, contains the highest density of cones in the human ${ }^{2.3 .4}$ or monkey retina (rev in $\left.{ }^{4.5,6}\right)$. Although the primate foveola is generally regarded to lack rods ${ }^{7}$, recent evidence indicates that the monkey can have rods within $50 \mu \mathrm{m}$ of the centre ${ }^{5.8}$, but the human does not have rods across a centralmost zone of $350 \mu \mathrm{m}^{3}$. Foveal circuitry is so constructed that each cone signals via several bipolar cells to at least two foveal ganglion cells (rev. in ${ }^{9.10}$ ), creating the neuronal substrate for high visual acuity and colour vision".

How does such a complex region develop? Previous work has shown that the fovea is specified very early in gestation. It is the first region to cease cell division which

Departments of Biological Structure and Opthalmology, University of Washington, Seattle, Washington 98195.

Correspondence to: Dr Anita Hendrickson, Dept. of Biological Structure, SM-2(), University of Washington, Seattle, Washington 98195.

Eye (1992) 6, 136-144 occurs by fetal day (Fd) 56-60 in monkey ${ }^{12}$ and around fetal week (Fwk) 14 in humans ${ }^{13}$. A morphological region containing only cones is identifiable by Fwk 10-12 14,15 . The foveal pit appears by Fd1 $25^{16}$ or Fwk $32^{17.18}$, apparently as a result of the peripheral-ward migration of GC first, followed later by neurons of the inner nuclear layer (INL). Late in gestation the photoreceptor layer changes, such that cones increase in density, elongate and thin, and rods come to lie progressively closer to the foveola ${ }^{16,18,19,20}$. This process has been ascribed to a central-ward migration of the photoreceptors during which the centralmost cones remodel to pack maximally into a much smaller area. Foveal maturation appears to be a slow process. despite the fact that it is the first retinal region to begin maturation. Foveal pit formation is not complete until well after birth in humans ${ }^{17.18}$. Human foveal cones are only beginning to form inner and outer segments at birth ${ }^{18}$ and adult cone density is not reached until after one year in monkeys ${ }^{20}$ and four years in humans ${ }^{19}$.

The immature morphological state of the human fovea has prompted speculation that the relatively low visual acuity of the human neonate may be due in large part to this immaturity ${ }^{21,22,23}$. However, it is difficult to test this hypothesis experimentally in humans. The Old World Macaca monkeys species ( $M$. nemestrina, M. mulatta) have been widely used to study normal visual system development, and have been particularly valuable as experimental models of various abnormal developmental conditions such as amblyopia or strabismus ${ }^{24,25.26 .27}$. When postnatal visual acuity development is compared in humans and $M$. nemestrina, the age-related curves are highly comparable if monkey weeks are equal to human months ${ }^{24}$. However, it is not clear if their retinal development is equally comparable, particularly for retinal morphology at birth. Morphological studies of prenatal $M$. nemestrina or $M$. mulatta ${ }^{16}$ indicate that foveal development is relatively advanced at birth, although cone density is still quite low compared to adult ${ }^{20}$. Therefore in this paper we have reanalysed our histology collection of $M$. nemestrina and human retinas to make a direct tem- 


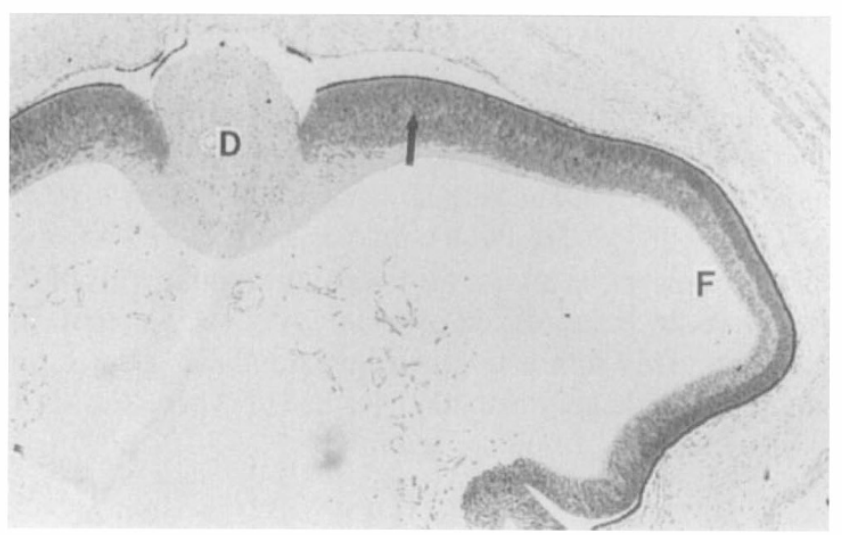

Fig. 1. Monkey retina at Fd60 showing the optic disc (D) and developing fovea $(F)$. The inner plexiform layer extends almost to the disc (arrow) in the nasal retina. The temporal retina peripheral to the fovea is very short and less mature than the nasal retina. Azure II-Methylene Blue stained plastic section; $50 X$.

poral comparison of their prenatal and postnatal foveal development.

\section{MATERIAL AND METHODS}

All monkey retinas were obtained from fetuses that were the result of timed pregnancies with a precision of $1-3 d \pm$.

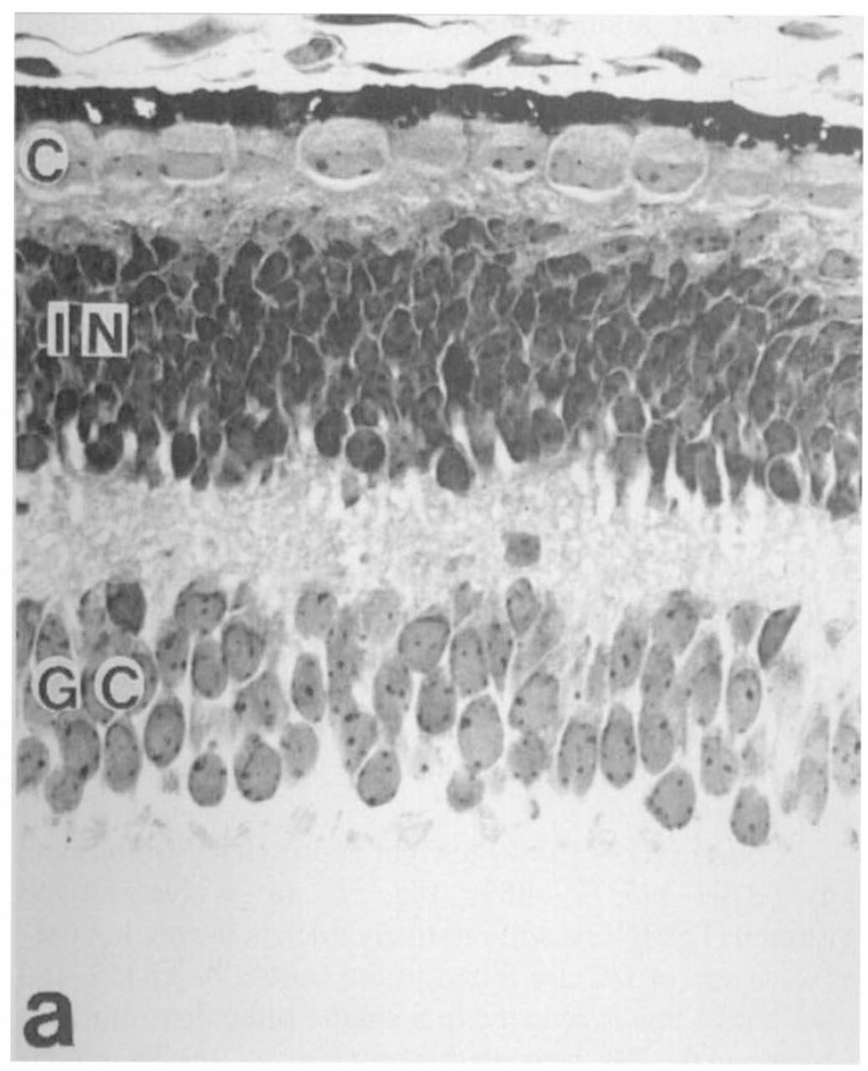

Fetal animals were delivered by aseptic caesarian section, given an overdose of barbituate and immediately perfused intravascularly. Postnatal animals were given an overdose of barbiturate and similarly perfused. The eyes were removed, and postfixed at least overnight. Fixatives included $4 \%$ paraformaldehyde (para), $4 \%$ para containing $0.1-5 \%$ glutaraldehyde (glut), $4 \%$ para/ $0.05-0.1 \%$ glut $/ 0.2 \%$ picric acid, or $2 \%$ para/ $2 \%$ glut; all fixatives were buffered with $0.1 \mathrm{M}$ phosphate at $\mathrm{pH} 7.4$. The horizontal meridian was cut from the globe and, in older animals, cut into a temporal portion containing disc, a variable amount of nasal retina, and temporal retina from disc to periphery. The retina was dehydrated, embedded in glycol methacrylate, serially sectioned at $2 \mu \mathrm{m}$ and every third section mounted for staining by $10 \%$ methylene blue-azure II.

Human retinas were obtained through the Lions' Eye Bank at the University of Washington. Eyes were enucleated within three hours of death, the anterior portion removed and the posterior half immediately immersed in $4 \%$ para $/ 0.5 \%$ glut. Eyes were then processed as above. Eyes with preexisting conditions of disease or injury that involved the retina were eliminated from this study. A brief medical history for most of the infants is given in ${ }^{18}$.

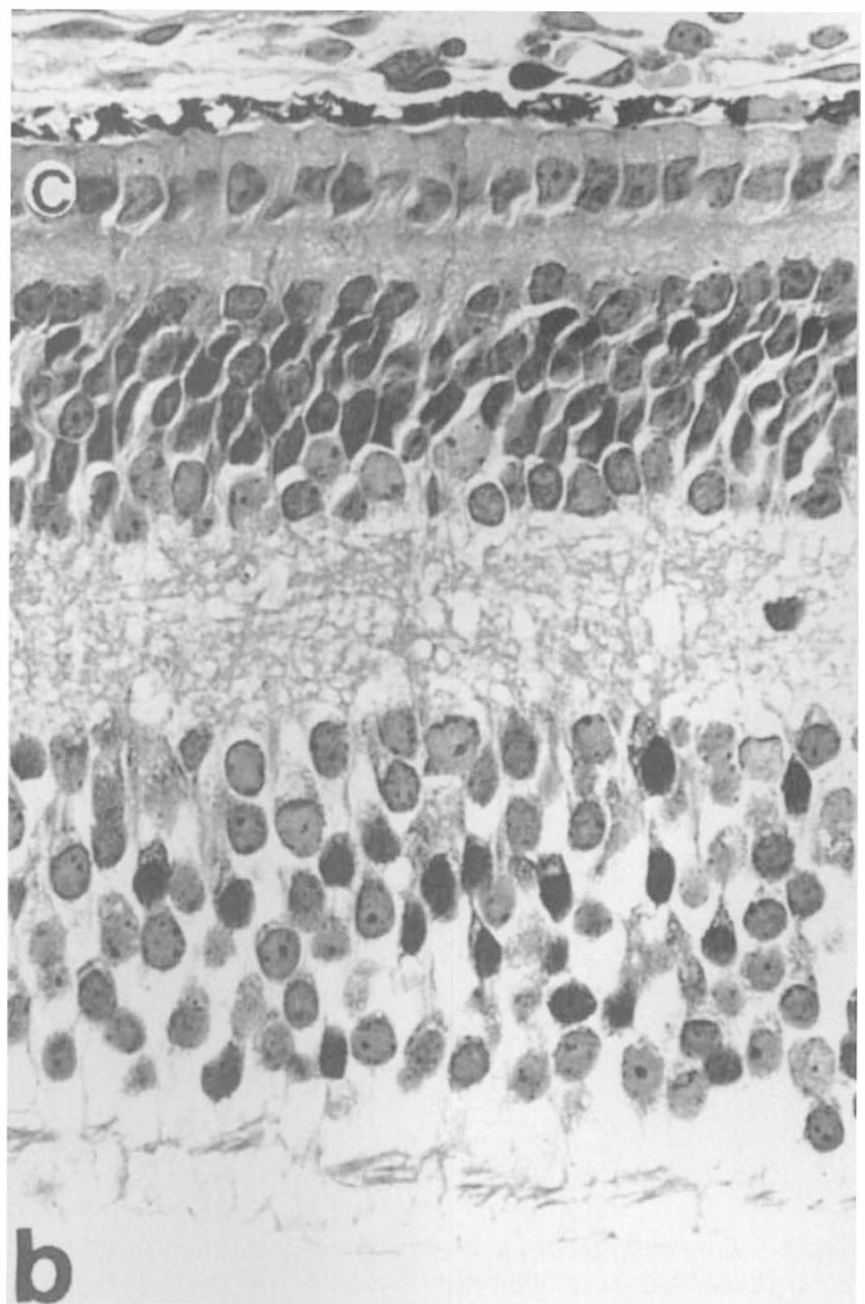

Fig. 2. Monkey fovea at $F d 60$ (a) and $F d 91$ (b) showing the increase laminar thickness and morphological maturation of the cones (C). The ganglion cell (GC) and inner nuclear (IN) layers are indicated in a. Azure II-Methylene Blue stained plastic section; 480X. 


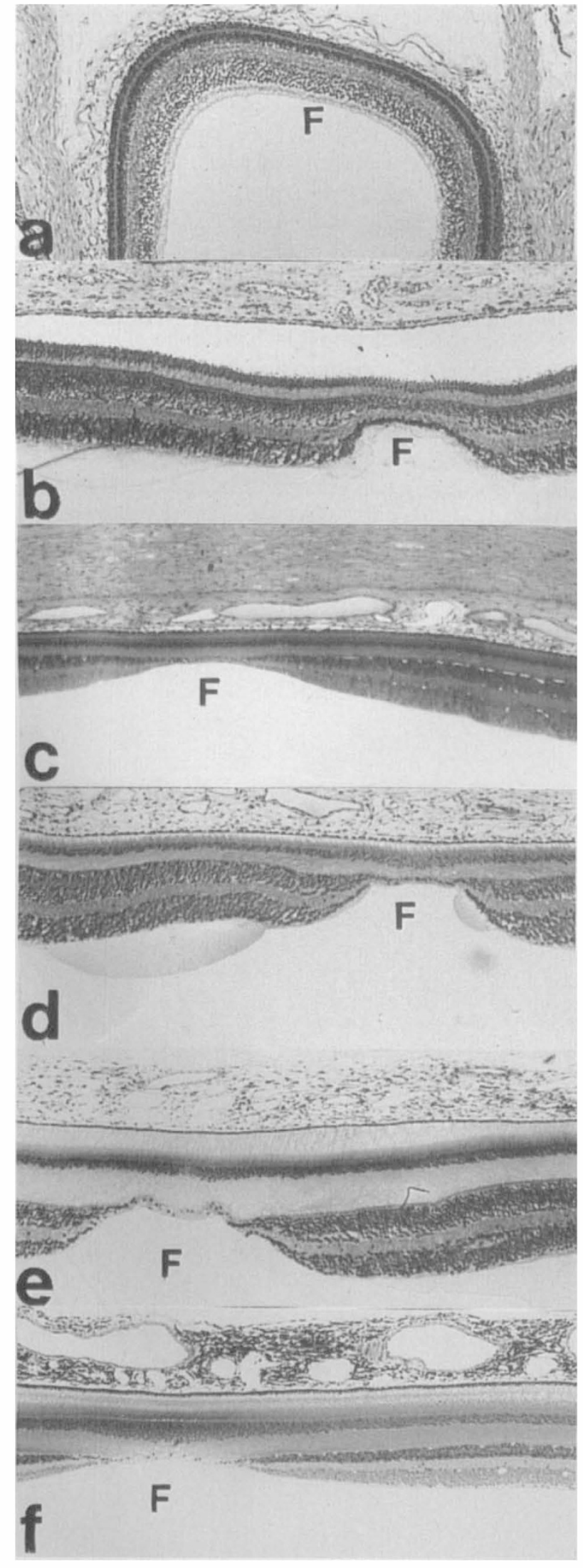

Fig. 3. Low magnification photomicrographs illustrating the maturational sequence of monkey fovea at $F d 83$ (a), Fd147 (b), Fd165 (c), Pld (d), P2wks (e) and P39wks (f). The foveal centre is indicated by an F. Azure II-Methylene Blue stained plastic sections; $50 X$.
Monkey retinas were examined at $\mathrm{Fd} 51,61,73,75,80$, $83,90,110,125,131,139,147,152,155,165$. Birth in the UW Regional Primate Research Center colony occurs on Fd166-170. Postnatal (P) ages included P1d; 2, 4, 6, 12 and 39 weeks. Human retinas were studied at Fwk 10.5, 20-21, 24-26, 32, 36, and P1 and 5d; 2, 11, 15, 36, 45, and 156 mo (13 yrs). Human (40Fwk) and monkey (168Fd) gestation can be corrected by expressing each as percent gestation. This figure is given in parenthesis after each actual prenatal age. Postnatal ages use the correction of 1 monkey week $=1$ human month $^{24}$.

\section{RESULTS}

\section{Monkey Foveal Development}

The region of the future fovea could be identified at Fd51 $(30 \%)$ as a small zone containing a packed layer of GC, a distinct inner plexiform layer (IPL), and a INL separated from a single layer of cones by a thin outer plexiform layer (OPL). This zone is indicated by an $F$ in Fig. 1. At $30 \%$ gestation this is the only region of retina that has three neuronal layers. It is actually thinner than surrounding retina, and characteristically is very tightly apposed to and flattened against the overlying pigment epithelium (PE). The IPL does not quite reach the disc nasally (Fig. 1, arrow) and only extends a short distance into the retina temporal to the fovea, suggesting a faster maturation in nasal retina. The foveal relationship to the disc remains constant throughout later development so that the prenatal fovea always lies about $3.7 \mathrm{~mm}$ temporal from the disc ${ }^{20}$.

By Fd60 (36\%) the adult layers of the fovea are present and some neuronal types can be identified (Fig. 2a). The GC layer is four to five cells deep and is separated from the INL by a relatively thick IPL; the IPL now extends nasally to the disc. The OPL is more clear and horizontal cells can be distinguished at its lower border. The cones (Fig. 2a, C) form a single line of round large cells with a slight apical predominance of cytoplasm. The retina is tightly apposed to the PE and no interphotoreceptor space is apparent. There is relatively little gross change up to Fd90 (53\%; Fig. 2b, 3a) except that the retinal layers get thicker, the cones are more tightly packed and they have elongated so that now the apical bulge might be called an inner segment (IS). Muller cell cytoplasmic processes are much darker than the cones, and run across the OPL to terminate at the external limiting membrane. The foveal pit is not yet apparent.

Around Fd110 (65\%) a slight depression develops and by Fd131-147 (77-88\%; Fig. 3b, 4a) a clear pit has formed. The INL is still relatively thick at the pit, but only 1-2 layers of GC are found in the centre. At Fd152-165 (92-98\%) this is reduced to a single, often discontinuous layer and the INL is much thinner (Fig. 3c, 4b). During the latter half of gestation cone morphology changes considerably. Cone packing density increases as evidenced by the increase in the number of cone nuclei in the foveal centre accompanied by a concomitent decrease in IS size. Between Fd90-125 (53-74\%) the cones become tilted 

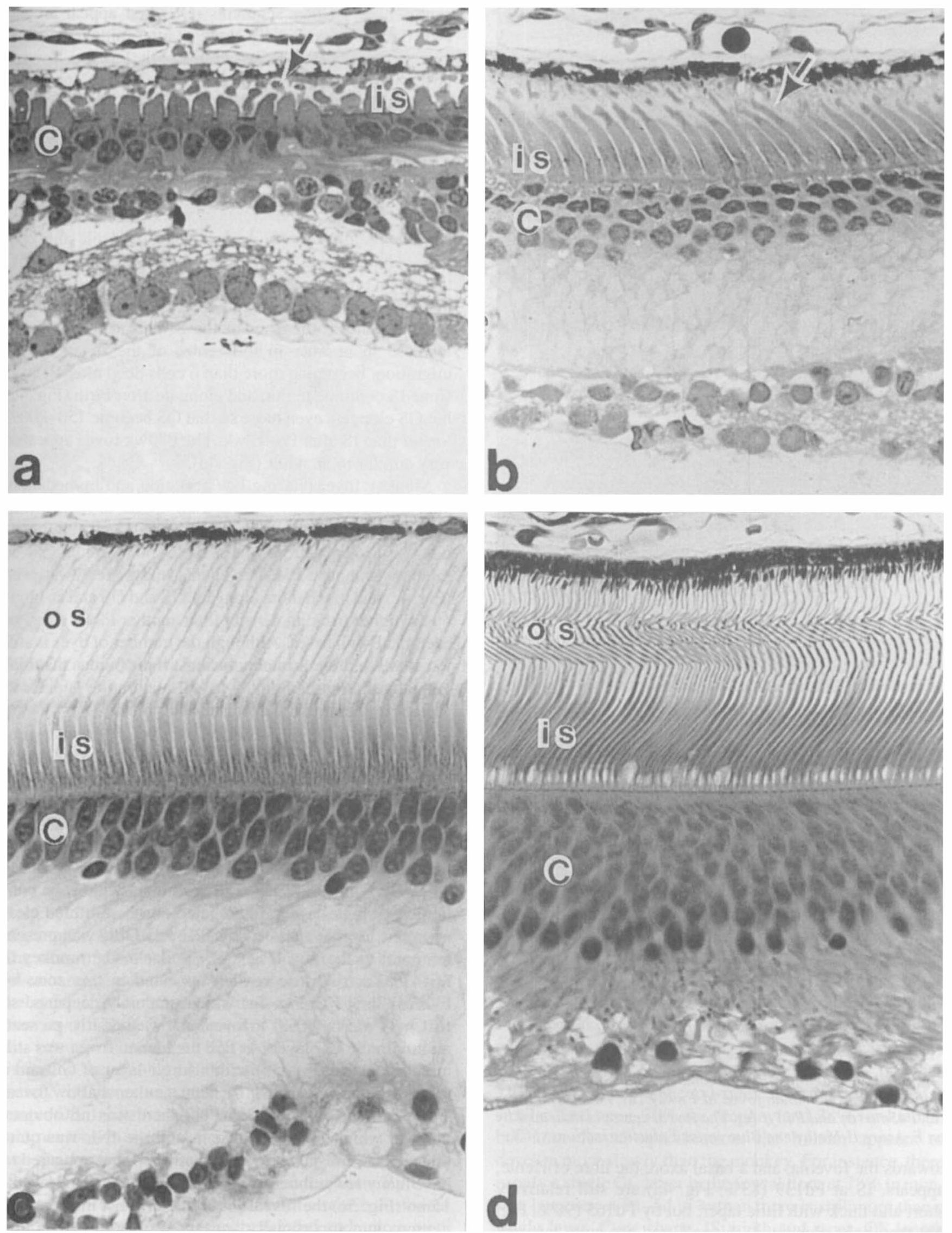

Fig. 4. Morphological maturation of cones $(C)$ in the centre of the developing monkey fovea at $F d 139$ (a), Fd165 (b), P2wks (c) and P39wks (d). Note the elongation of the inner segments (IS) and the growth of the outer segments (arrows in $\mathbf{a}, \mathbf{b}$ and OS in $\mathbf{c}, \mathbf{d})$. A single layer of inner retinal neurons remains in a-c, but their number decreases with age. Azure II-Methylene Blue stained plastic sections; $480 X$. 


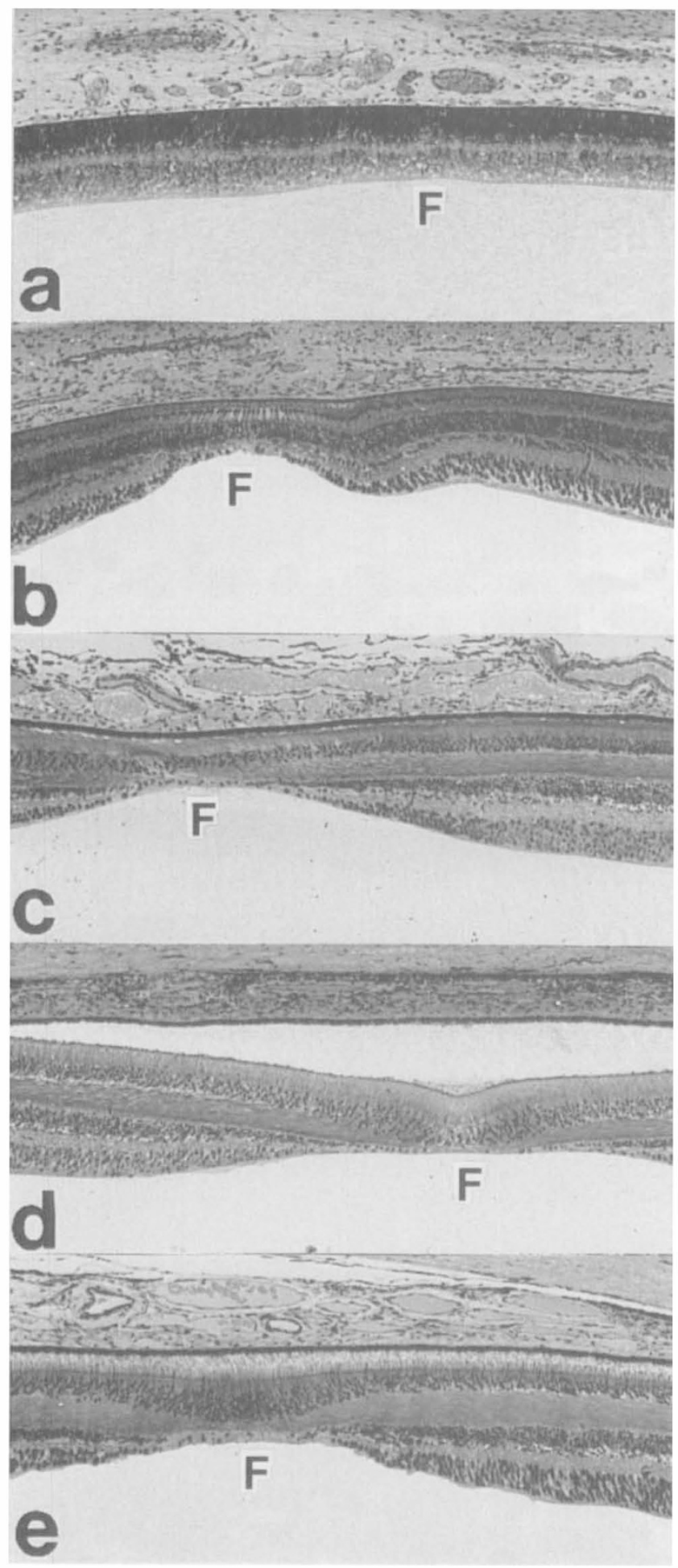

Fig. 5. Low magnification photomicrographs illustrating the maturation of the human fovea at Fwk24 (a), P5d (b), P15mo (c), P45mo (d) and Pl3yr (e). The foveal centre is indicated by an F. Azure II-Methylene Blue stained plastics sections; 50X.

towards the foveola, and a basal axon, the fibre of Henle, appears. IS at Fd139 (83\%; Fig. 4a) are still relatively short and thick with little taper, but by Fd165 (98\%; Fig. $4 \mathrm{~b}$ ) they have elongated, thinned, and are quite tapered. Cone nuclei become three to four deep in the foveola around birth (Fig. 3c, d), indicative of accelerated migration of slightly more peripheral cones into the foveal centre $^{16,18,19,20}$. Outer segments (OS) first appear around Fd110 (65\%), and rapidly elongate after midgestation, so that by birth OS are $30-50 \%$ of IS length (Fig. 4b). Toward birth the fibres of Henle become increasingly prominent and the OPL becomes much thicker, due both to the elongation of individual axons and to an increase in total axon number as cones migrate into the foveal centre.

After birth (Fig. 3d, e) inner retinal neurons continue to migrate laterally so that by $\mathrm{P} 2-4 \mathrm{wk}$ only a single discontinuous layer of cells remains in the pit and the foveola consists mainly of cone nuclei, fibres of Henle and glial cells. This process continues so that by P39wk (Fig. 3f) the foveal pit has a prominent slope formed by 6-8 layers of GC and an even thicker INL, and only scattered neurons can be found in the floor of the foveal pit. Cone nuclei increase in number in the centre of the fovea due to migration, becoming more than 6 cells deep after P12wk. Cone IS continue to thin and elongate after birth (Fig. 4c) but OS elongate even more so that OS become 150-200\% longer than IS after P6-12wk. The P39wk fovea appeared very similar to an adult (Fig. 4d).

Monkey foveas during late gestation and immediately after birth varied somewhat in their degree of maturity. In an earlier study ${ }^{16}$ which included several paraffin-embedded eyes around Fd155F, the same foveal morphology was found over about a $10 \mathrm{~d}$ span. In this study, one P2wk fovea (Fig. 4c) had much more elongated IS and OS and an obviously higher packing density than another P2wk fovea or even the P4wk fovea. Although the number of eyes is still too small to draw firm conclusions, it is obvious that biological variation in monkey foveal maturation is present, at least in the month before and after birth.

\section{Human Foveal Development}

At Fwk10.5 (26\%) the human eye closely resembles the monkey at $30 \%$ (Fig. 1), except that it is larger overall. The future fovea consists of a narrow zone $500-700 \mu \mathrm{m}$ wide containing only cones, a very narrow OPL and a rather ragged IPL. Mitosis are still present on the edges of this zone. By Fwk 21-22 (53\%) a prominent wide zone containing a single layer of elongated cones and three clear neuronal layers separated by IPL and OPL was present temporal to the disc (Fig. 6a), similar to the monkey at $36-47 \%$. A slight depression appeared in this zone by Fwk24-26 (63\%; Fig. 5a) which gradually deepened so that by FwK36 (90\%) a foveal pit was clearly present, mainly in the GC layer. At P5d the human fovea was still quite immature (Fig. 5b) with a single layer of GC and a relatively-unchanged INL forming a rather shallow fovea. The P1d fovea was swollen, but there was no obvious point at which GC were absent, and the INL was quite thick. The P2mo fovea was also swollen, but appeared to have many fewer inner retinal neurons in the pit. By P11$15 \mathrm{mo}$ (Fig. 5c) the foveal pit is free of most inner retinal neurons and superficially appears similar to the $\mathrm{P} 45 \mathrm{mo}$ (Fig. 5d) or P156mo fovea (Fig. 5e).

Maturation of foveal cones in the human follows the same course as that described in the monkey, but over a 

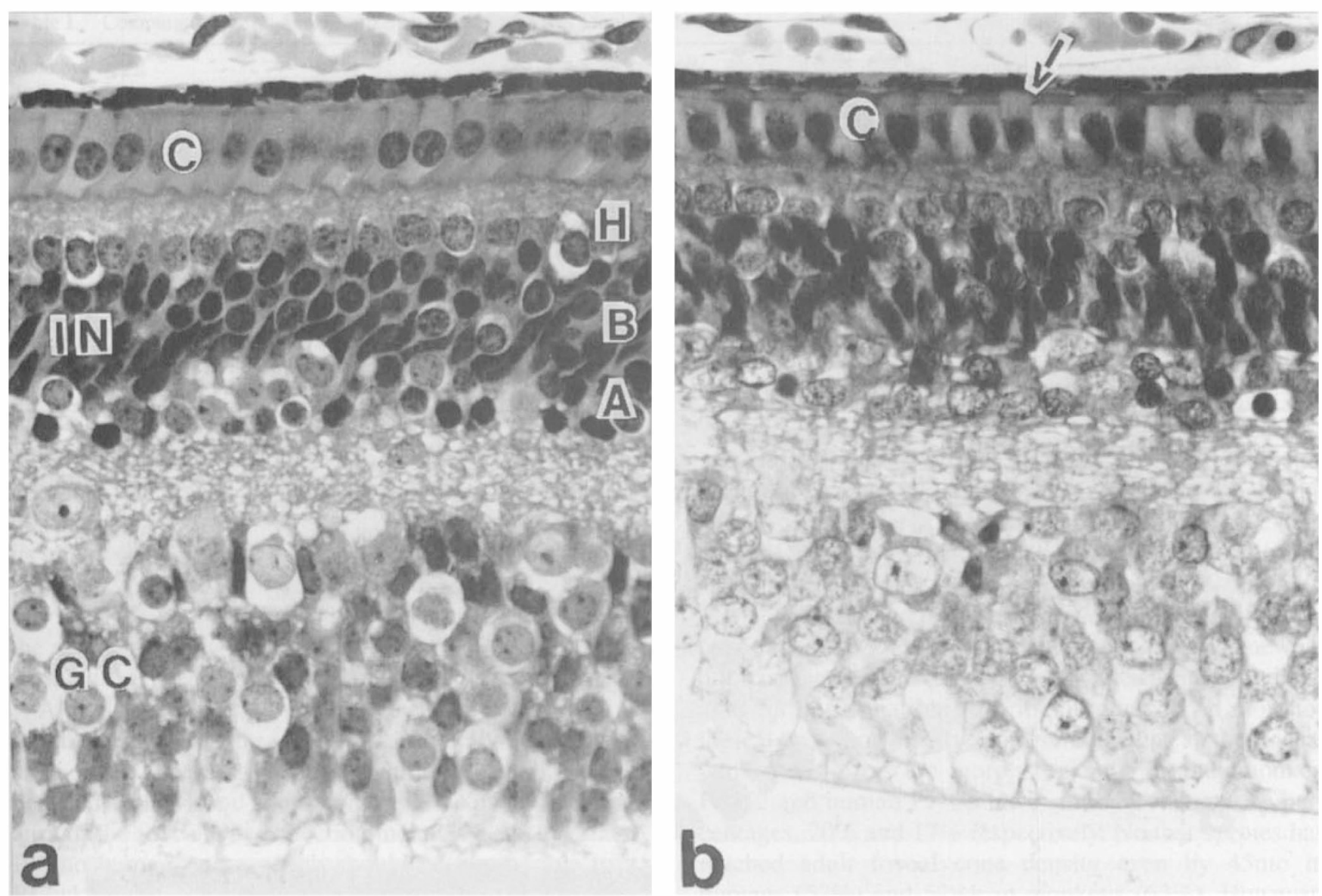

Fig. 6. Central fovea in human retina at $F w k 21$ (a) and $F w k 24$ (b) showing the formation of inner segments (arrow in b) and the fiber of Henle on the cones $(C)$ by $F w k 24$. The ganglion cell $(G C)$ and inner nuclear (IN) layers are indicated in a. Horizontal (H), bipolar (B) and amacrine (A) cells can be distinguished at both ages in the IN layer. Azure II-Methylene Blue stained plastic sections; 480X.

longer time. By Fwk 24-26 (63\%; Fig. 6b) cones have a small IS and a short fibre of Henle and are even more elongated by Fwk36 (90\%) when tiny OS can be seen. Human cones in the first postnatal week (Fig. 7a) still form a single row of dark cells separated by pale Müller cell cytoplasm, suggesting that relatively little centralward migration has occurred by this age. Each centralmost foveal cone has a fibre of Henle which ends in a synaptic pedicle immediately below each cone, the IS are thick and short with a slight taper, and each cone has a short OS (Fig. 7a, arrow). The fibre of Henle is longer and angled away from the fovea centre on cones near the rod-cone border zone, and the synaptic pedicle on these cones is displaced laterally from its cell body ${ }^{18}$. The retina of the P5d baby (Fig. 7a) was better preserved than that of the P1d, but, within this limitation, morphology of the cones seemed to be approximately the same, except that the P1d baby had slightly longer OS. In contrast, the P5d baby had fewer inner retinal neurons in the foveal pit. The P2mo infant's retina was poorly preserved, but it could be seen that foveal cone IS, OS and fibres of Henle continue to lengthen. By P11$15 \mathrm{mo}$ (Fig. 7b) only scattered neurons remain in the foveal floor, foveal cones are markedly thinned and enlongated, and foveal cone density is much increased, as evidenced by the clustering of cone nuclei in the foveola. This process continues so that by P45mo (Fig. 7c) cones are four to six nuclei deep and almost all inner retinal neurons have migrated laterally so that the foveal floor mainly contains fibres of Henle and glial processes. Cone density probably increases further with age, because the foveolar cones at P13yr (Fig. 7d) are packed more tightly and IS and OS are much thinner and longer.

\section{DISCUSSION}

This temporal comparative survey of the morphology of the developing monkey and human fovea is summarised in Table I. To facilitate prenatal comparison, the two different gestational periods have been corrected by expressing fetal age as percent gestation, and then plotting when the same foveal developmental changes are found. Postnatal ages have been corrected by assuming that one monkey postnatal week is equal to one human postnatal month ${ }^{24}$. The fovea, defined as the rod-free zone, can be identified at similar fetal ages, $30 \%$ for monkey and $28 \%$ for human, but after foveal specification, the human appears to develop more slowly than the monkey. For instance, there is only a single GC layer in the foveal floor at $78 \%$ in monkey, but even at P1-5d in human there is still more than a single layer. Cone shape, IS width and taper, OS length and fibre of Henle length are all relatively mature at birth in monkey, but still markedly immature in the first postnatal week in both neonatal human infant retinas. In fact, 

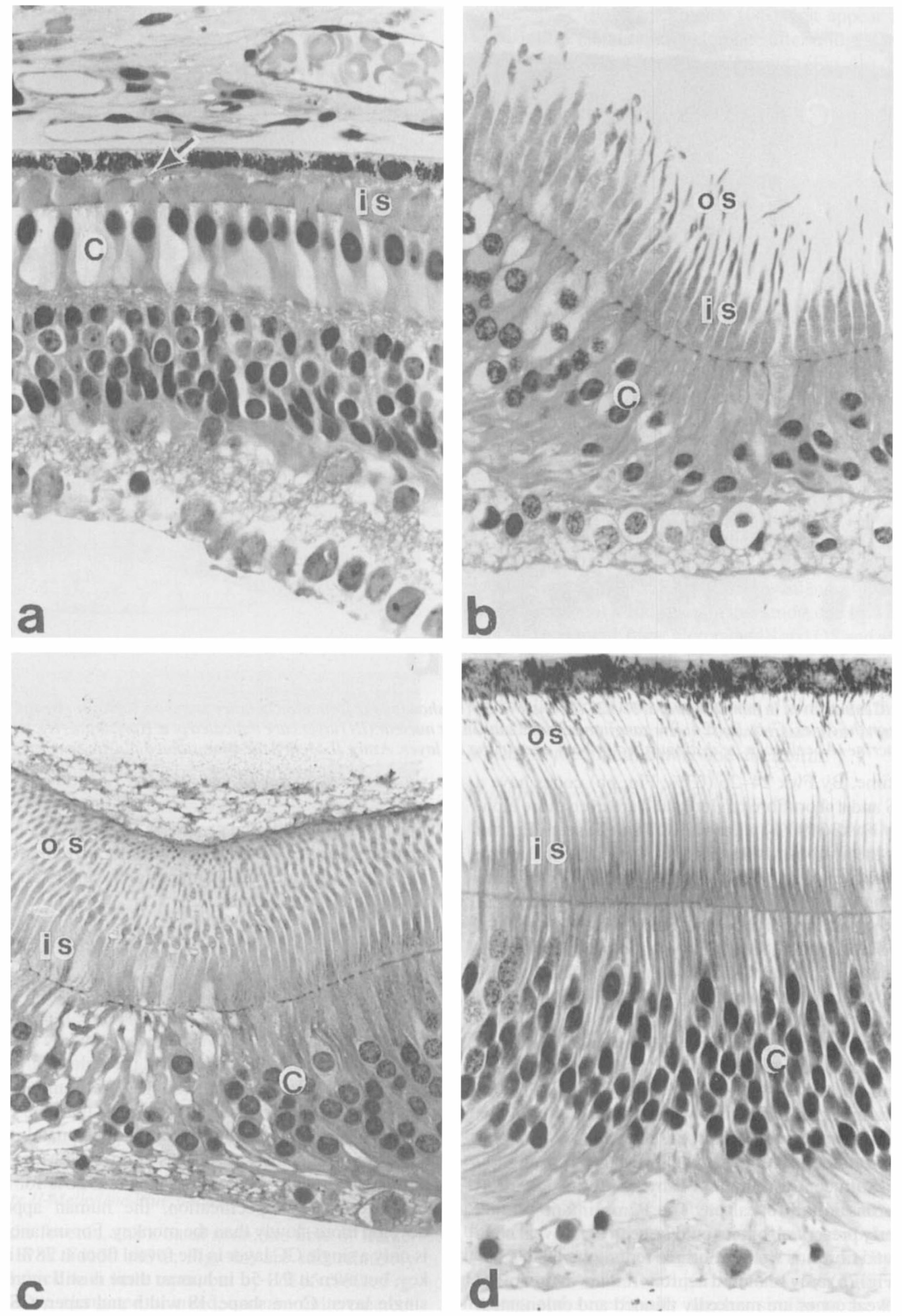

Fig. 7. Postnatal maturation of central foveal cones $(C)$ in human retina $t$ P5d (a), P11mo (b), P45mo (c) and P13yr (d). Note the elongation of the inner segments (IS), increased packing of $C$ nuclei and growth of outer segments (arrow in a, OS in b-d) Azure IIMethylene Blue stained plastic sections; $480 \mathrm{X}$. 
Table I. Comparative Development of Foveal Morphology in Monkey and Human

\begin{tabular}{|c|c|c|}
\hline $\begin{array}{l}\text { Monkey } \\
\text { Birth = } 168 \mathrm{~d}\end{array}$ & Foveal Morphology & $\begin{array}{l}\text { Human } \\
\text { Birth }=40 \mathrm{wk}\end{array}$ \\
\hline $30 \%$ & Cones form a continuous row; OPL present & $26 \%$ \\
\hline $55 \%$ & Cones tilt toward fovea centre, tibres of Henle appear & $63 \%$ \\
\hline $65 \%$ & Shallow foveal pit in GCL: OS appear & $6.3 \%$ \\
\hline $78-88 \%$ & $\begin{array}{l}\text { GCL } 1-2 \text { cells thick; INL thinned; single layer cones elongated with thick, tapered IS and } \\
\text { clear OS }\end{array}$ & $\mathrm{Pl}-5 \mathrm{~d}$ \\
\hline $92-98 \%$ & $\begin{array}{l}\text { GCL/INL fused in foveola: cones } 3 \text { deep; IS thinner, elongated and tapered; OS } 30-50 \% \\
\text { of IS length }\end{array}$ & P2mo? \\
\hline $2-6 w k$ & Scattered GCL/INL layer; very elongated IS: OS $100 \%+$ of IS length & $11-15 \mathrm{mo}$ \\
\hline $12 w k$ & Cones $4-5$ deep; OS $150 \%$ IS; IS much thinner; foveola mainly cone axons and glial cells & $45 \mathrm{mo}$ \\
\hline $39 w k$ & Nearly adult cone density; scattered GCL/INL layer; OS 200\% IS & $1.56 \mathrm{mo}$ \\
\hline
\end{tabular}

even the $\mathrm{P} 2 \mathrm{mo}$ human cones were less mature than a newborn monkey, although the morphological preservation of this human eye was not optimal which may have obscured its true maturational state. Based on morphology found in this study, the newborn human fovea is similar to a monkey fovea at Fd131-155 or 78-88\% gestation. Therefore, the neonatal monkey fovea is not morphologically comparible to the neonatal human fovea.

Although clearly the human fovea begins postanatal life at a relatively immature stage, it develops more rapidly after birth. There is relatively little gross difference between a P12wk and adult monkey in the overall appearance of the fovea. Our collection includes a Pl lmo and a P15mo human retina which should be comparible to a P12wk monkey. These human foveas have a well developed pit, although cone packing density, IS and OS width and length, and the thickness of the fibre of Henle layer all are somewhat less mature than a P45mo human. Thus we have concluded earlier ${ }^{18}$ that the human fovea generally has an adult appearance by $\mathrm{P} 15 \mathrm{mo}$, and it appears that the monkey also has a mature fovea at a similar time of $\mathrm{P} 12 \mathrm{wk}$. Individual cone morphology, however, is more similar in Pl2wk monkeys and P45mo humans, suggesting that the outer retina in humans develops somewhat more slowly postnatally.

Cone packing density is another measure of maturity. It has been determined in both species in our laboratory, although by different methods ${ }^{18.20}$. Foveal cone density development is compared in Table II by determining the percent of adult density at corrected fetal ages and comparible postnatal ages. Although more ages must be counted to draw more definite conclusions, and several animals at the same age need to be counted to determine age-related variability, this comparison shows that both monkey and human cone density rises in a regular fashion from late gestation to late infancy. Monkey has somewhat higher cone densities than human in late gestation and just after birth, compatible with its more advanced morphological development. It is interesting that at the age at which the foveas are morphologically similar, monkey Fd152 and human Pl-5d have similar cone density percentages, $20 \%$ and $17 \%$ respectively. Neither species has reached adult foveal cone density even by $45 \mathrm{mo}$ in humans $(52 \%)$ and $52 \mathrm{wk}$ in monkeys $(63 \%)$. However, note that the P26wk monkey was $75 \%$ adult cone density; either this animal was precocious or the P60wk was somewhat retarded. If the P26wk monkey is typical, then the human develops more slowly postnatally as well. From this trend, we might predict that $M$. nemestrina would reach adult densities around P72wk (18mo), but the human may not be fully adult until after P6yr.

This study has shown that overall foveal development including cone density increase is qualitatively and quantitatively similar in monkeys and man. Our major finding is that monkey fovea appears to be more mature than human at birth. In contrast, because both species reach similar stages by P11-15mo/wk, this means that human has an accelerated postnatal development. Experimental

Table II. Comparative Development of Foveal Cone Density in Monkey and Human

\begin{tabular}{|c|c|c|c|}
\hline \multicolumn{2}{|c|}{ Monkey' } & \multicolumn{2}{|c|}{ Human² } \\
\hline Age & $\%$ adult density & Age & $\%$ adult density \\
\hline & & $53 \%$ & $9 \%$ \\
\hline & & $63 \%$ & $11 \%$ \\
\hline $90 \%$ & $20 \%$ & & \\
\hline $98 \%$ & $41 \%$ & & \\
\hline & & P5d & $17 \%$ \\
\hline P6wk & $31 \%$ & & \\
\hline Pl3wk & $39 \%$ & $\mathrm{P} 15 \mathrm{mo}$ & $25 \%$ \\
\hline P26wk & $75 \%$ & & \\
\hline & & $\mathrm{P} 4.5 \mathrm{mo}$ & $52 \%$ \\
\hline P52wk & $63 \%$ & & \\
\hline
\end{tabular}

'data modified from 20 .

'data modified from 19. 
lid suture in monkeys has not found any change in qualitative or quantitative aspects of foveal development ${ }^{28}$, nor was the fovea changed after neonatal atropinisation ${ }^{29}$ or dark rearing ${ }^{30}$. However, neonatal aphakia ${ }^{31}$ and eyelid suture $^{32}$ do alter neurotransmitter expression in monkey retina, and if this is correlated with age, it could be expected that human retina would be even more severely affected. This study has shown that the M. nemestrina fovea is a good model for human if sequential age is taken into account. However, the relative immaturity of the human retina at birth and its rapid development over the early postanatal years means that it may be more susceptible to nutritional, toxic, environmental or deprivation influences during early post-natal development.

This work was supported by EY04536, EY01208 and in part by Research to Prevent Blindness, Inc., Vision CORE EY07013 and RR00166 to the Regional Primate Center at the University of Washington. The human eyes were obtained through the invaluable help of the Lions' Eye Bank at the University of Washington. The technical assistance of Aurora Guardiano, Roshen Hendrickson and Criss Meligro is gratefully acknowledged.

Key words: Development, Fovea, Human, Monkey, Retina.

\section{REFERENCES}

1. Curcio CA and Allen KA: Topography of Ganglion Cells in Human Retina. J comp Neurol 1990, 300: 5-25.

2. Osterberg GA: Topography of the layer of rods and cones in the human retina. Acta Opthalmol 1935, 13: 1-94.

3. Curcio CA, Sloan KR, Kalina RE, Hendrickson A: Human photoreceptor topography. J comp Neurol 1990, 292: 497-523.

4. Curcio CA and Hendrickson A: Organization and development of the primate photoreceptor mosaic. In Osborne NN and Chader GJ eds. Progress in Retinal Research, vol. 10. Oxford, Pergamon Press, 1991: 90-120.

5. Packer O, Hendrickson AE, Curcio CA: Photoreceptor topography of the adult pigtail macaque (Macaca nemestrina) retina. J comp Neurol 1989, 288: 165-83.

6. Wickler KC, Williams RW, Rakic P: Photoreceptor mosaic: number and distribution of rods and cones in the rhesus monkey retina. J comp Neurol 1990, 297: 499-508.

7. Polyak SL: The Retina. Chicago, Univ Chicago Press, 1941.

8. Borwein B, Borwein D, Medeiros J, et al: The ultrastructure of monkey foveal photoreceptors, with special reference to the structure, shape, size and spacing of the foveal cones. Am J Anat 1980, 159: 125-46.

9. Schein S: Anatomy of macaque fovea and spatial densities of neurons in foveal representation. J comp Neurol 1988, 269: 479-505.

10. Wässle H, Grunert U and Rohrenbeck J: Cortical magnification factor and the ganglion cell density in the primate retina. Nature 1989, 341: 643-6.

11. Kaplan E, Lee BB, Shapley RM: New Views of Primate Retinal Function. In Osborne NM and Chader GJ eds. Progress in Retinal Research, vol. 9. Oxford, Pergamon Press, 1990.

12. La Vail MM, Rapaport DH and Rakic P: Cytogenesis in the monkey retina. J comp Neurol 1991, 309: 86-114.

13. Provis JM, van Driel D, Bilson FA, Russell P: Development of the human retina: Patterns of cell distribution and redistribution in the ganglion cell layer. J comp Neurol 1985, 233: 429-51.

14. Hollenberg MJ and Spira AW: Early development of the human retina. Canad J Ophthalmol 1972, 7: 472-91.

15. Linberg KA and Fisher SK: A burst of differentiation in the outer posterior retina of the eleven-week human fetus: an ultrastructural study. Vis Neurosci 1990, 5: 43-??.

16. Hendrickson $A$ and Kupfer $C$ : The histogenesis of the fovea in the macaque monkey. Invest Ophthalmol 1976, 15: 746-56.

17. Mann I: The Development of the Human Eye, 3rd ed. New York, Grune and Stratton, 1969.

18. Hendrickson AE and Youdelis C: The morphological development of the human fovea. Ophthalmology 1984, 91: 603-12.

19. Yuodelis $\mathrm{C}$ and Hendrickson A: a qualitative and quantitative analysis of the human fovea during development. Vision Res 1986, 26: 847-56.

20. Packer O, Hendrickson AE and Curcio CA: Developmental redistribution of photoreceptors across the Macaca nemestrina (pigtail macaque) retina. J comp Neurol 1990, 298: 472-93.

21. Banks MS and Bennett PJ: Optical and photoreceptor immaturities limit the spatial and chromatic vision of human neonates. J opt Soc Am A 1988, 5: 2059-80.

22. Brown AM, Dobson V, Maier J: Visual acuity of human infants at scotopic, mesopic and photopic luminances. Vision Res 1987, 27: 1845-58.

23. Wilson HR: Development of spatiotemporal mechanisms in infant vision. Vision Res 1988, 28: 611-28.

24. Boothe RG, Dobson V, Teller DY: Postnatal development of vision in human and nonhuman primates. Ann Rev Neurosci 1985, 8: 495-545.

25. LeVay S, Wiesel TN, Hubel DH: The development of ocular dominance columns in normal and visually deprived monkeys. J comp Neurol 1980, 191: 1.

26. Kiorpes L, Boothe RG, Hendrickson AE, Movshon JA, Eggers HM, Gizzi MS: Effects of early unilateral blur on the macaque's visual system. I. Behavioral observations. $J$ Neurosci 1987, 7: 1318-25.

27. Harwerth RW, Smith EL III, Crawford MLF, von Noorden GK: Behavioral studies of the sensitive periods of development of visual functions in monkeys. Behav Brain Res 1990, 41: 179-98.

28. Clark D, Hendrickson A, Curcio C: Photoreceptor topography in lid-sutured Macaca fascicularis. Invest Ophthalmol Vis Sci (Suppl); 1988. 29: 33.

29. Hendrickson AE, Movshon JA, Eggers HM, Gizzi MS, Boothe RG, Kiorpes L: Effects of early unilateral blur on the macaque's visual system. II. Anatomical observations. $J$ Neurosci 1987, 7: 1326-39.

30. Hendrickson AE and Boothe R: Morphology of the retina and dorsal lateral geniculate nucleus in dark reared monkeys (Macaca nemestrina). Vision Res 1971, 15: 517-21.

31. Iuvone PM, Tigges M, Fernandes A, Tigges J: Dopamine synthesis and metabolism in rhesus monkey retina: development, aging, and the effects of monocular visual deprivation. Vis Neurosci 1989, 2: 465-72.

32. Stone R, Laties A, Raviola $E$ and Wiesel T: Increase in retinal vasoactive intestinal polypeptide after eyelid fusion in primates. Proc Nat Acad Sci USA 1988, 85: 257-60. 